\title{
GESTÃO DA COMUNICAÇÃO E GESTÃO DO CONHECIMENTO EM PROJETOS COMPLEXOS: UMA ANÁLISE DA LITERATURA
}

\author{
Jeniffer de Nadae \\ jnadae@gmail.com \\ Diego Clemente \\ diego_hclemente@hotmail.com \\ Graziela Galvão \\ graziela.galvao@gmail.com \\ Marly Carvalho \\ marlymc@usp.br
}

A complexidade dos projetos pode intensificar o papel da comunicação e do conhecimento da equipe devido ao aumento da necessidade de coordenação e tomada de decisão. Assim, o objetivo deste trabalho é analisar as publicações sobre comunicação, conhecimento em projetos complexos, por meio de uma revisão sistemática da literatura e bibliometria. Para a revisão sistemática da literatura buscou-se artigos utilizando os termos communication* and complex* and project* and knowledge" na base de dados da Web of Science, permitindo analisar os principais autores sobre o tema, tendências de pesquisa e gaps na literatura que possibilitarão desenvolvimento de futuras pesquisas que contribuirão com o tema. Nota-se que a literatura engloba estudos sobre gestão da comunicação nos times dos projetos, mais precisamente sobre armazenagem e compartilhamento do conhecimento em projetos de TI, saúde, física, química, construção, entre outros. Poucos estudos focam em projetos complexos, principalmente analisando os dois tópicos conjuntamente. Destaca-se a ausência de artigos, na base utilizada, que analisam as barreiras à comunicação em projetos e barreiras à gestão do conhecimento em projetos, principalmente analisando estas barreiras nos projetos complexos, não foram encontrados estudos que fazem esta relação. Assim, com base nos artigos analisados existem poucos estudos que relacionam gestão da comunicação e gestão do conhecimento em projetos e nenhum artigo que relaciona gestão da comunicação e gestão do conhecimento em projetos complexos. Individualmente os temas são estudados por diversos autores, como apresentados nas tabelas e analises deste estudo.

Palavras-chave: comunicacao, Conhecimento, Complexo, projeto, Bibliometria 


\section{Introdução}

O gerenciamento da complexidade do projeto é um fator crítico que afeta o sucesso do projeto (ALLEN, 2008; BACCARINI, 1996). A complexidade do projeto geralmente está relacionada à forma como o sistema do projeto é modelado Vidal e Marle (2008), assim, diferentes classificações de projetos complexos são apresentadas na literatura por (Baccarini, 1996; Benbya e McKelvey, 2006; Carvalho, Patah e Bido, 2015; Chapman e Hyland, 2004; Cicmil e Marshall, 2005; Cooke-Davies et al., 2008; Crawford, Hobbs e Turner, 2005; Eriksson et al., 2002; Geraldi e Adlbrecht, 2007; Geraldi, Maylor e Williams, 2011; Green, 2004; He et al., 2015, 2012; Hobday, 1998; Homer-Dixon, 2000; IPMA - Internacional Project Management Association, 2006; Kim e Wilemon, 2003; Little, 2005; Luo et al., 2017; Müller e Turner, 2007; Mykytyn e Green, 1992; Santos et al., 2016; Shenhar, 2001; Shenhar e Dvir, 2007; Shenhar, Dvir e Shulman, 1995; Tatikonda e Rosenthal, 2000; Vidal e Marle, 2008; Williams, 2005, 1999; Xia, 2005) estes autores propõem dimensões que avaliam a complexidade do projeto.

A complexidade dos projetos pode intensificar o papel da comunicação e do conhecimento da equipe devido ao aumento da necessidade de coordenação e tomada de decisão (Marks, Mathieu e Zaccaro, 2001).

Para os autores Rabechini Jr., Carvalho e Laurindo (2002) para atingir uma comunicação eficaz, é necessário um processo de gerência do sistema de comunicação que no estágio de maior abrangência, é chamado de gestão do conhecimento. Já Alonso et al. (2013) indicam que uma vez que o conhecimento é transmitido principalmente por meio de relações humanas, quaisquer ruídos geram distorções na informação, criando barreiras à comunicação e dificultando o armazenamento e compartilhamento do conhecimento, criando então as barreiras à gestão do conhecimento. Assim, pode-se afirmar que muitas das barreiras presentes na transferência de conhecimento são de comunicação Fukuyama et al. (2015) e o mesmo também pode ocorrer.

Diante disso, o objetivo deste trabalho é analisar as publicações sobre comunicação, conhecimento em projetos complexos, por meio de uma revisão sistemática da literatura e bibliometria. Para a revisão sistemática da literatura buscou-se artigos utilizando os termos

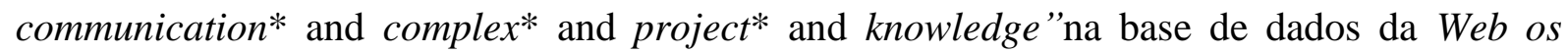
Science, permitindo analisar os principais autores sobre o tema, tendências de pesquisa e gaps 
na literatura que possibilitarão desenvolvimento de futuras pesquisas que contribuirão com o tema.

Para a consecução do proposto este trabalho está dividido em 5 seções. A primeira contextualiza o tema abordado. Uma breve apresentação dos conceitos é apresentada na seção 2, assim como o método de pesquisa, etapas da busca na base de dados do Web of Science, os softwares e redes de análise são apresentados na seção 3. Os resultados e a discussão sobre o tema são apresentados na seção 4 e as conclusões na seção 5.

\section{Gestão da comunicação e gestão do conhecimento em projetos complexos}

O processo de comunicação é influenciado por vários fatores ambientais, incluindo localização, iniciador, relação de poder, tamanho e composição do grupo, disposição física, finalidade e tempo (JOHANSEN e GILLARD, 2005). As barreiras podem ocorrer por diversos fatores, tais como: conflitos, ambientes culturais distintos, ausência de feedback, censura na informação e canais inadequados (CARVALHO e RABECHINI JR, 2015). (ALENCAR, SOUZA e VIANA, 2008) reforçam que é preciso eliminar quaisquer tipos de barreiras que dificultem a comunicação ou impeçam a transmissão e o entendimento claro e objetivo da mensagem.

A distribuição geográfica das Equipes; Comunicação e coordenação; Sistemas integrados; Processos de negócios e estruturas de comunicação também podem representar barreiras à comunicação (BANO, ZOWGHI e SARKISSIAN, 2016).

Johannessen e Olsen (2011) enfatizam a importância da comunicação em projetos, particularmente aqueles que são grandes e complexos. Eles argumentam que as empresas devem passar do uso dos processos de comunicação para o uso das capacidades de comunicação durante os projetos.

O compartilhamento do conhecimento pode ser visto como os processos de transferir conhecimento de pessoas, grupos ou organizações, que podem incluir informações, ideias e habilidades relevantes (LEE, 2001). No que diz respeito ao compartilhamento de conhecimento em projetos complexos, há duas dimensões de conhecimento a serem abordadas: (1) conhecimento sobre o objeto do projeto, ou seja, o produto a ser desenvolvido e as especificações técnicas que levam a um projeto que atenda aos requisitos, tais como 
peças, componentes, peças ou montagens, e/ou tecnologias utilizadas; (2) conhecimento necessário para executar o projeto, isto é, estrutura de trabalho e gerenciamento, horários, equipes e habilidades envolvidas, conhecimento, sistemas e interfaces científicos e técnicos relevantes (SANTOS et al., 2016).

Cicmil (2005) oferece uma estrutura conceitual para capturar conhecimento, propondo cinco aspectos do conhecimento em projeto: contexto; conteúdo; comportamento organizacional; comunicação e congruência de projetos.

A complexidade do projeto é propriedade de um projeto que dificulta a compreensão, a previsão e a manutenção do seu comportamento geral, mesmo quando há informações razoavelmente completas sobre o sistema do projeto. Os fatores relacionados ao tamanho do projeto, variedade de projetos, interdependência do projeto e contexto do projeto podem auxiliar a dimensionar a complexidade de um projeto (VIDAL e MARLE, 2008).

Diferentes classificações de projetos complexos são apresentadas na literatura. Baccarini, (1996) considera a complexidade do projeto através dos conceitos de complexidade tecnológica e complexidade organizacional. Enquanto que Shenhar, Dvir e Shulman (1995) distinguem duas dimensões da complexidade do projeto: "incerteza tecnológica" e "escopo do sistema". Shenhar (2001) amplia o quadro para abranger três dimensões da complexidade do projeto: "incerteza", o "ritmo" e a "complexidade/escopo" onde o "ritmo" é adicionado para refletir "velocidade e criatividade" dos objetivos de tempo

A gestão de um projeto complexo envolve várias fases diferentes, às vezes sobrepostas. Muitos dos métodos, técnicas e ferramentas disponíveis de gerenciamento de projetos disponíveis são aplicados na gestão de um projeto complexo. Na prática, isso significaria que mais de $60 \%$ dos elementos de competência de projetos podem ser aplicados nos projetos complexos (IPMA - INTERNACIONAL PROJECT MANAGEMENT ASSOCIATION, 2006).

\section{Materiais e métodos}

Devido ao objetivo da pesquisa, uma Revisão Sistemática da Literatura (RSL) foi realizada para ajudar a responder às seguintes questões de pesquisa: Como a literatura relaciona gestão da comunicação e gestão do conhecimento em projetos complexos? e Quais são as principais 
tendências na literatura acadêmica sobre a gestão da comunicação e a gestão do conhecimento em projetos complexos?

Para obter um panorama geral da literatura sobre o tema, foi consultada a base de dados Web of Science, pois os artigos publicados em periódicos indexados e com fator de impacto calculado pelo Journal Citation Report (JCR) são localizados nesta base.

Os procedimentos foram organizados em cada etapa do protocolo de pesquisa Littell; Corcoran; Pillai (2008), seguindo três etapas: coleta de dados, análise de dados e síntese. (TRANFIELD; DENYER; SMART, 2003).

A etapa de coleta de dados foi realizada em maio de 2018, a busca utilizou os termos: "communication* and complex* and project* and knowledge" a base de dados selecionou os títulos, resumos e palavras-chave com os termos indicados e resultou em 80 artigos, selecionando apenas artigos em português e inglês a base final contempla 41 artigos (Tabela $1)$.

Tabela 1 - Critérios de busca na base de dados

\begin{tabular}{|l|l|c|}
\hline $\begin{array}{l}\text { Title, Abstract and } \\
\text { Keyword }\end{array}$ & $\begin{array}{l}\text { communication* and } \text { complex }^{\star} \text { and } \text { project }^{*} \text { and } \\
\text { knowledge }\end{array}$ & $\mathbf{8 4 7}$ \\
\hline $\begin{array}{l}\text { Tipo de documento } \\
\text { Idioma }\end{array}$ & $\begin{array}{l}\text { Article e Review } \\
\text { Inglês e português }\end{array}$ & $\mathbf{4 1 1}$ \\
\hline Mês da Busca & Maio/ 2018 & \\
\hline
\end{tabular}

Fonte: Os autores

Após selecionar os 411 artigos, conduziu-se a etapa de análise de dados, fundindo bibliometria e revisão sistemática da literatura.

A análise da rede foi realizada utilizando os seguintes softwares: VosViewer que gerou a rede de citação de palavras-chave, citação dos principais autores. Além disso, com a base de dados realizou-se uma análise descritiva, gerando gráficos, por meio do excel dos países com maior quantidade de publicações sobre o tema, evolução de pesquisas ao longo dos anos, principais periódicos que publicam sobre o tema, os autores mais citados, outliers com o total de 
citações por autores. Na sequência, a análise de conteúdo foi realizada, quando os artigos pesquisados foram classificados e codificados.

A análise das palavras chave é útil para evidenciar conceitos associados à questão de pesquisa, dando maior consistência à discussão conceitual de desenvolvimento sustentável e indicadores de desempenho associados a essa temática. Em seguida, a análise de conteúdo foi conduzida com base na leitura de resumo e da última seção de cada artigo, denominados como "discussões", “conclusões", "resultados", entre outros (LOCKE; GOLDEN-BIDDLE, 1997). A escolha dessa parte do artigo se dá pelo fato de que esse conteúdo muitas vezes traz um resumo breve do artigo e, além disso, contém os principais resultados da pesquisa. Isso permite identificar a contribuição de cada artigo, bem como lacunas de conhecimento.

Finalmente, o terceiro passo, a síntese da bibliometria foi conduzida. De acordo com Crossan e Apaydin (2010), este é o passo em que é adicionado mais valor a análise bibliométrica, pois é onde existe a criação de novos conhecimentos com base na análise completa e minuciosa dos dados. Insights emergentes das duas etapas anteriores trouxe o material necessário para construir um modelo conceitual por meio de um processo indutivo.

\section{Resultados e discussões}

A pesquisa resultou em uma base de 411 artigos, com média de citações por item de 15,43 e h-index de 39. Iniciando a análise dos artigos pela quantidade de publicações por ano (Figura 1), nota-se que a primeira publicação surgiu em 1991, sendo que os anos de 2013 e 2017 tem uma somatória maior de artigos sobre o tema. O primeiro artigo (ano de 1991) tratava da relação entre a comunicação e o conhecimento em projetos. Os artigos datados do ano de 2013 versam em sua maioria sobre gestão do conhecimento: softwares de transferência do conhecimento, conhecimento como fator critico de sucesso, a relação entre o conhecimento, aprendizagem e informação em projetos complexos e projetos de Tecnologia de Informação (TI).

Os artigos de 2017 em sua maioria tratam dos temas de conhecimento e aprendizagem, confiança e comunicação, projetos complexos em TI e na área da saúde, sustentabilidade em projetos e a influencia do conhecimento e da comunicação nas mudanças ambientais dos projetos. 
Figura 1 - Publicações por ano e citações em cada ano

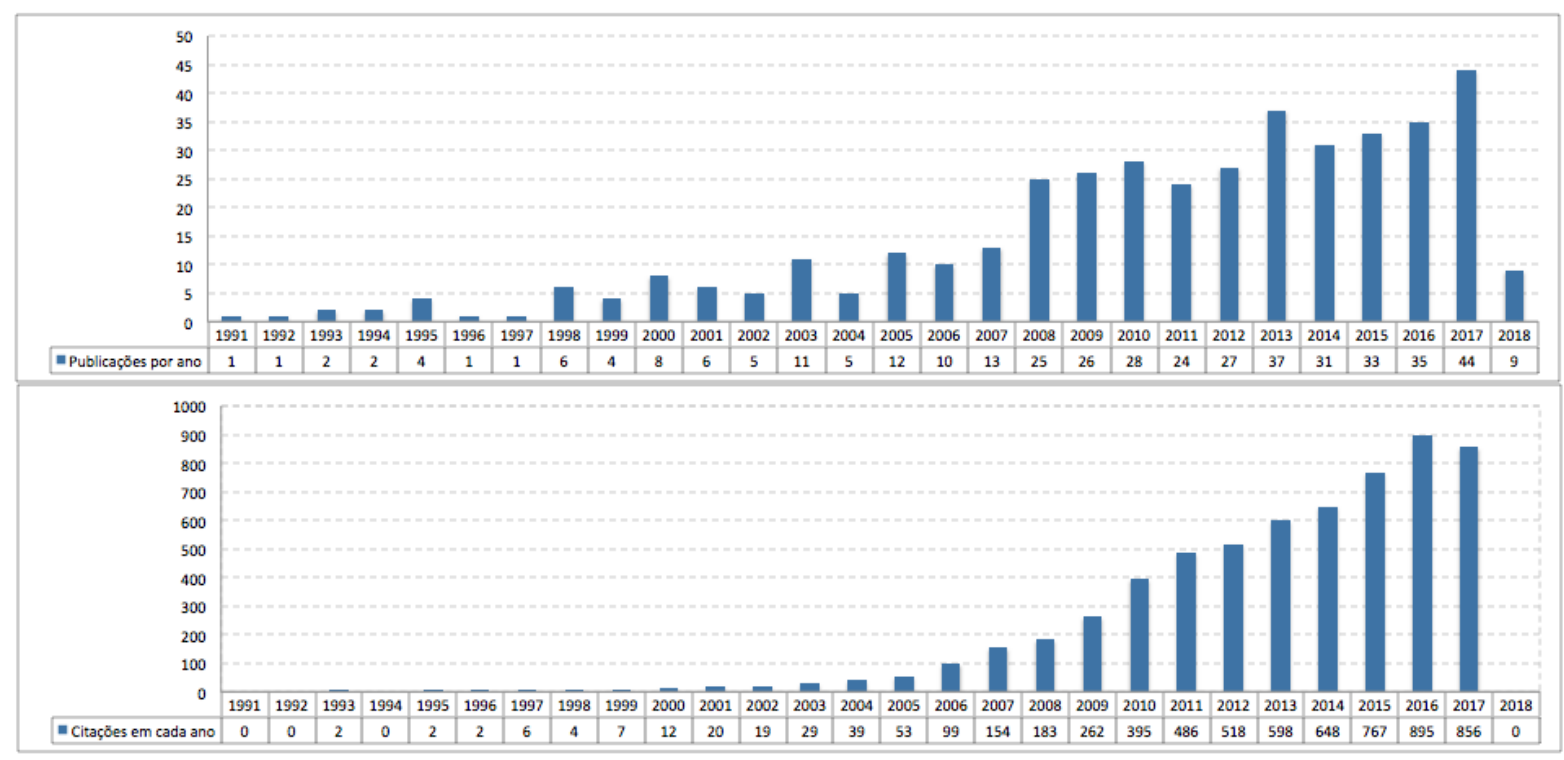

Nota: As publicações foram selecionadas até o mês de maio de 2018

Fonte: Os autores

Nota-se que as citações dos artigos em cada ano acompanham a evolução das publicações sobre o tema.

Os periódicos com mais de duas publicações sobre o tema são os apresentados na Tabela 2, os demais periódicos apresentam apenas uma publicação sobre o tema, conforme destacado na tabela.

Tabela 2- Título dos periódicos 


\begin{tabular}{|c|c|c|c|}
\hline Periódicos & \% Total & Periódicos & \% Total \\
\hline Journal Of Management In Engineering & 1.946 & IDS Bulletin Institute Of Development Studies & 0.487 \\
\hline International Journal Of Project Management & 1.703 & IEEE Transactions On Communications & 0.487 \\
\hline Automation In Construction & 1.217 & IEEE Transactions On Industrial Informatics & 0.487 \\
\hline Computers Education & 1.217 & Industrial Management Data Systems & 0.487 \\
\hline IEEE Transactions On Professional Communication & 0.973 & Information And Software Technology & 0.487 \\
\hline Implementation Science & 0.973 & Information Management & 0.487 \\
\hline Lecture Notes In Artificial Intelligence & 0.973 & Information Systems Journal & 0.487 \\
\hline Project Management Journal & 0.973 & International Journal Of Engineering Education & 0.487 \\
\hline $\begin{array}{l}\text { Ai Edam Artificial Intelligence For Engineering Design } \\
\text { Analysis And Manufacturing }\end{array}$ & 0.730 & International Journal Of Information Management & 0.487 \\
\hline Computers In Human Behavior & 0.730 & Journal For Nature Conservation & 0.487 \\
\hline Computers In Industry & 0.730 & Journal Of Comparative Neurology & 0.487 \\
\hline Environmental Science And Pollution Research & 0.730 & Journal Of Digital Imaging & 0.487 \\
\hline IEEE Transactions On Engineering Management & 0.730 & Journal Of Evaluation In Clinical Practice & 0.487 \\
\hline IEEE Transactions On Wireless Communications & 0.730 & Journal Of Interprofessional Care & 0.487 \\
\hline International Journal Of Medical Informatics & 0.730 & Journal Of Management Information Systems & 0.487 \\
\hline Journal Of Engineering And Technology Management & 0.730 & Journal Of Medical Internet Research & 0.487 \\
\hline Journal Of Systems And Software & 0.730 & Journal Of Technology Transfer & 0.487 \\
\hline $\begin{array}{l}\text { Journal Of The American Society For Information } \\
\text { Science And Technology }\end{array}$ & 0.730 & Knowledge Management In Electronic Government & 0.487 \\
\hline Mis Quarterly & 0.730 & Kybernetes & 0.487 \\
\hline Research In Engineering Design & 0.730 & Lecture Notes In Computer Science & 0.487 \\
\hline Science Of The Total Environment & 0.730 & Organization Science & 0.487 \\
\hline American Journal Of Pharmaceutical Education & 0.487 & $\begin{array}{l}\text { Philosophical Transactions Of The Royal Society A } \\
\text { Mathematical Physical And Engineering Sciences }\end{array}$ & 0.487 \\
\hline Bmc Public Health & 0.487 & Preventive Medicine & 0.487 \\
\hline Concurrent Engineering Research And Applications & 0.487 & Quality Safety In Health Care & 0.487 \\
\hline Ecology And Society & 0.487 & R D Management & 0.487 \\
\hline Empirical Software Engineering & 0.487 & Sustainability & 0.487 \\
\hline Environmental Modelling Software & 0.487 & Technical Communication & 0.487 \\
\hline Environmental Research Letters & 0.487 & Technological Forecasting And Social Change & 0.487 \\
\hline Environmental Science Policy & 0.487 & Tehnicki Vjesnik Technical Gazette & 0.487 \\
\hline \multirow[t]{2}{*}{ European Physical Journal Special Topics } & 0.487 & Trials & 0.487 \\
\hline & & Outros* & 6.350 \\
\hline
\end{tabular}

Nota: mínimo dois artigos sobre o tema

Fonte: Os autores

Dentre os periódicos apresentados os que possuem maior quantidade de artigos publicados sobre o tema são Journal of Management in Engineering; International Journal of Project Management; Automation in Construction; e Computers Education. O periódico Journal of Management in Engineering tem fator de impacto de 2,011 tem em seu escopo estudos sobre questões de gestão e liderança incluem gerenciamento de contratos, gerenciamento de projetos, parcerias, gerenciamento de escritório, prática e desenvolvimento profissional, gestão financeira, gestão de recursos humanos, marketing e vendas, ética, tecnologia e inovação, gerenciamento de informações, educação continuada, teoria organizacional, planejamento estratégico, gestão de conflitos, negociação, gestão de riscos, globalização, networking, gestão de mudanças, trabalho em equipe, formação de equipes, comunicação, 
mentoria, coaching e diversidade, entre outros temas. O International Journal of Project Management tem fator de impacto 4,034, sendo um periódico publicado em colaboração com a Association for Project Management (APM) e a International Project Management Association (IPMA).

Já o Automation in Construction com fator de impacto de 2,919 e publica material referendado sobre todos os aspectos relativos ao uso de Tecnologias de Informação em Design, Engenharia, Tecnologias de Construção e Manutenção e Gestão de Instalações Construídas. O periódico Computers Education tem fator de impacto de 3,819 e visa aumentar o conhecimento e a compreensão das maneiras pelas quais a tecnologia digital pode melhorar a educação, através da publicação de pesquisas de alta qualidade, que ampliam a teoria e a prática. Percebe-se que o unico periódico com escopo voltado à àrea de gestão de projetos é o International Journal of Project Management, sendo ele também o que possui maior fator de impacto dentre os analisados.

Verificando as áreas com maior concentração de artigos sobre o tema, destacam-se as áreas de: Engenharia com aproximadamente $21,41 \%$ do total de artigos, seguidos pela área Ciências da Computação com 17,52\%, conforme Tabela 3. Nota-se que a quantidade de artigos se concentra na área de Engenharia e Computação e justificam-se pelo fato de que muitos estudos analisarem projetos de construção civil e projetos de TI.

Tabela 3 - Áreas de pesquisa com maior quantidade de artigos sobre o tema 


\begin{tabular}{lc}
\hline \multicolumn{1}{c}{ Áreas de pesquisa } & \% Total \\
\hline Engineering & 21.411 \\
Computer Science & 17.518 \\
Business Economics & 15.085 \\
Environmental Sciences Ecology & 10.949 \\
Education Educational Research & 7.786 \\
Health Care Sciences Services & 6.326 \\
Information Science Library Science & 6.326 \\
Public Environmental Occupational Health & 3.650 \\
Science Technology Other Topics & 3.650 \\
Psychology & 3.163 \\
Operations Research Management Science & 2.920 \\
Social Sciences Other Topics & 2.676 \\
Communication & 2.433 \\
Medical Informatics & 2.433 \\
Nursing & 2.433 \\
Telecommunications & 2.433 \\
Geology & 2.190 \\
Agriculture & 1.946 \\
Construction Building Technology & 1.946 \\
Public Administration & 1.946 \\
Neurosciences Neurology & 1.703 \\
General Internal Medicine & 1.460 \\
Geography & 1.460 \\
Meteorology Atmospheric Sciences & 1.460 \\
Water Resources & 1.460 \\
Marine Freshwater Biology & 1.217 \\
Pharmacology Pharmacy & 1.217 \\
Outras* & 1.217 \\
\hline \multicolumn{1}{c}{ * Corresponde a 65 áreas com menos de } \\
\end{tabular}

Fonte: Os autores

Os autores considerados outliers da amostra estão apresentados na Figura 2. Um outlier é uma observação atipicamente grande ou pequena que pode ter efeitos desproporcionais sobre os resultados estatísticos de uma amostra, como por exemplo a média, o que pode resultar em interpretações equivocadas (Minitab, 2017).

Para Figueira (1998), um outlier é caracterizado pela sua relação com as observações (dados) restantes que fazem parte da amostra. O distanciamento entre o outlier e essas observações é fundamental para sua correta caracterização. Os outliers são conhecidos também como observações anormais, contaminantes, estranhas, extremas ou aberrantes. A Figura 2 apresenta os autores outliers obtidos por meio do software Excel.

Figura 2 - Autores 


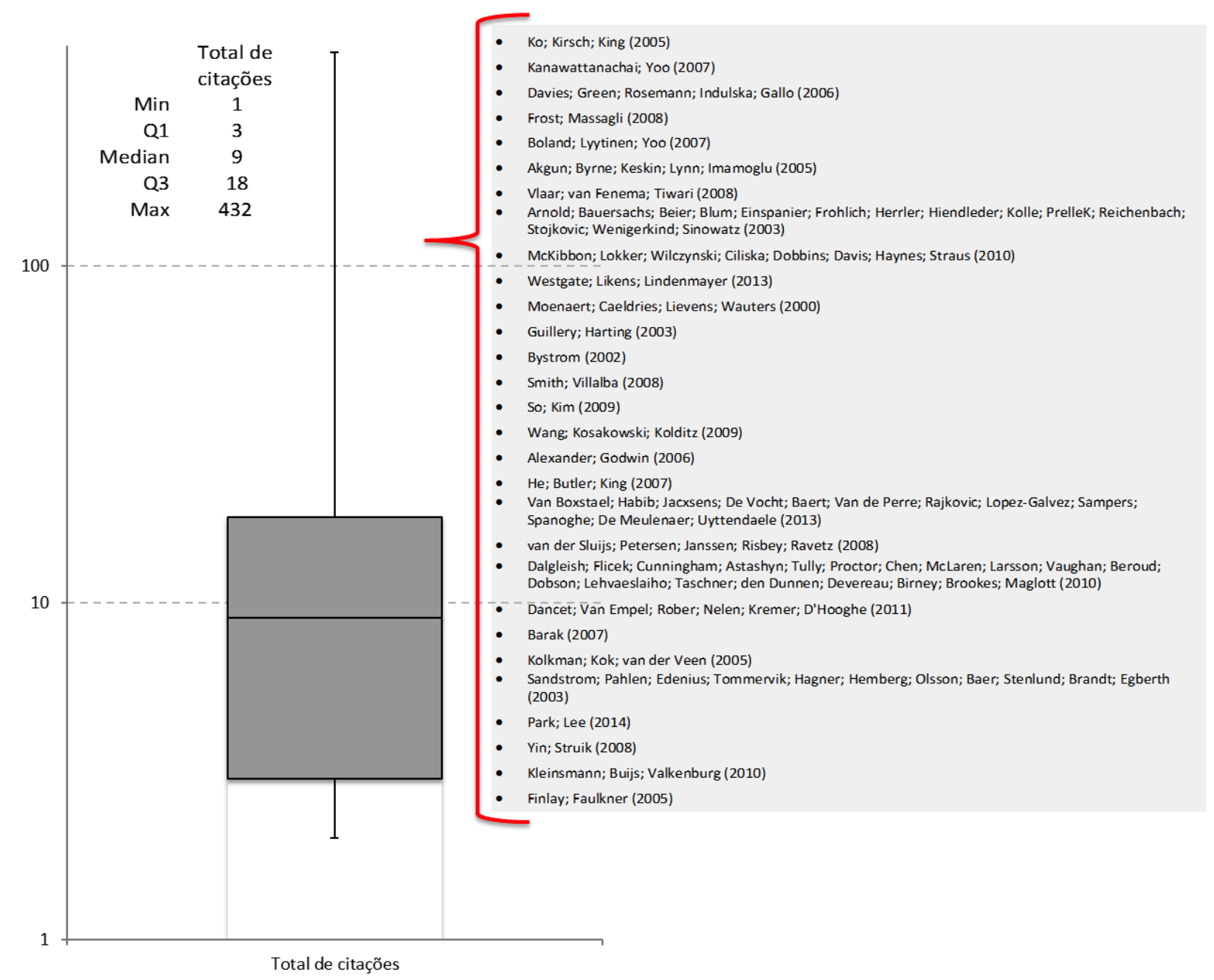

Fonte: Os autores

Optou-se por analisar os autores considerados acima da média de citações, os outliers extremos, sendo a mediana dos 411 artigos da amostra aproximadamente 9, portanto, os artigos com mais de 50 citações serão analisados com maior detalhe. Vale destacar que os autores considerados outliers extremos são Ko; Kirsch; King (2005) com 432 citações e média de 30,86 citações por ano assim, apresenta-se na Tabela 4 os autores mais citados e um resumo do escopo da pesquisa desenvolvida, total e média de citações dos artigos.

O mapeamento de palavras-chave e termos mais utilizados podem auxiliar pesquisadores na definiçãa dos tópicos de pesquisa em suas buscas e estudos. O software VOS Viewer oferece uma série de análises gráficas baseadas na co-ocorrência dos itens analisados (VAN ECK; WALTMAN, 2010). O sistema mostra a conexão entre termos e autores relacionados, 
proporcionando a divisão em grupos denominados clusters. Cada cluster é representado por uma cor e agrega todos os itens considerados similares.

O tamanho dos círculos dos mapas demonstra o número de ocorrência do item e a proximidade entre dois itens revela seu grau de relação, quanto mais próximos, tanto mais relacionados (VAN ECK; WALTMAN, 2010).

Quanto mais importante um item, tanto maior será a sua escrita e o seu círculo representativo (VAN ECK; WALTMAN, 2010). De acordo com a Figura 3 as palavras-chave que mais se destacam são: communication e knowledge, em seguida maanegment e performance. A rede de palavras-chave apresentam sete clusters.

Figura 3 - Co-ocorrência de todas as palavras-chave.

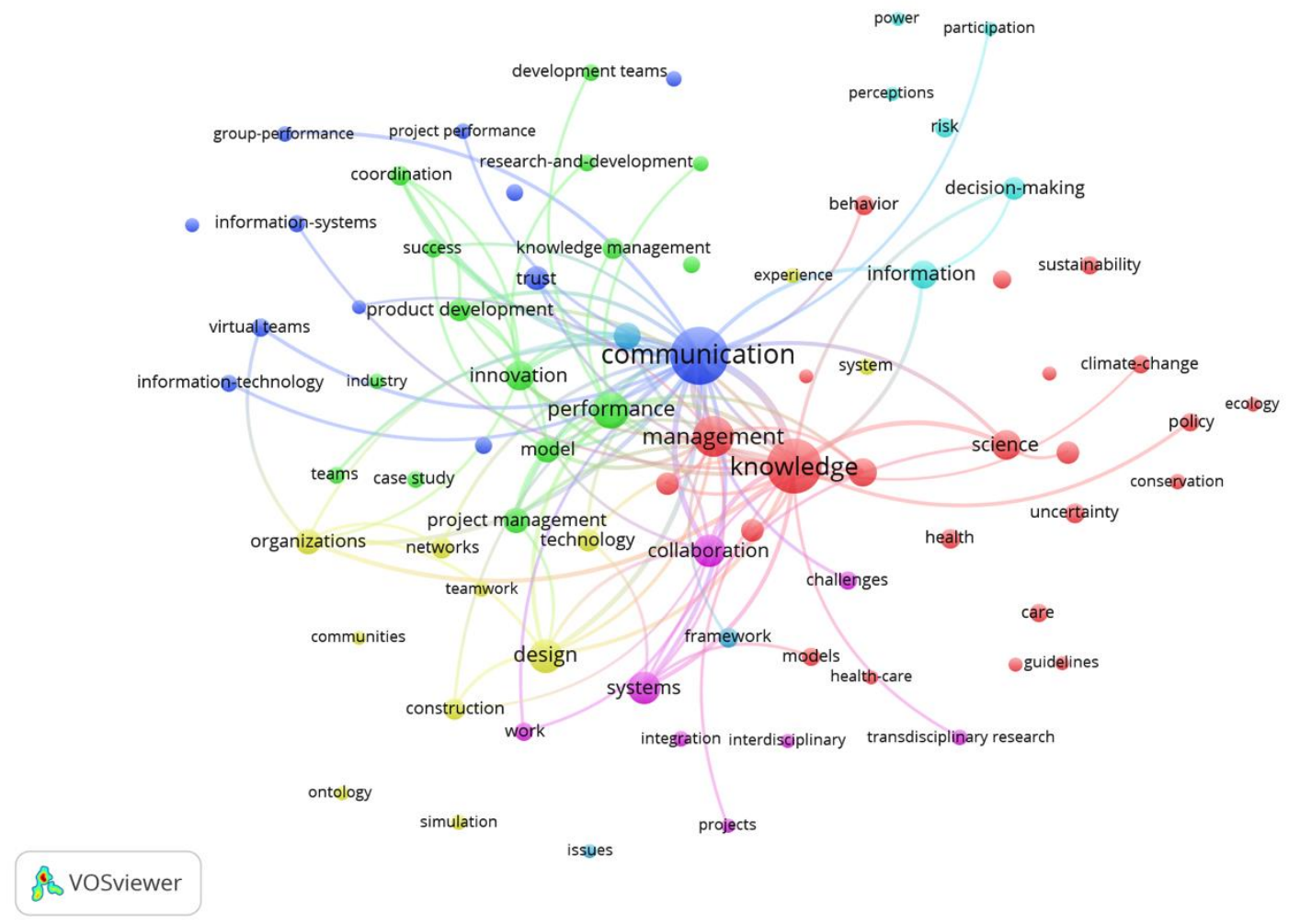

Nota: Esta rede foi realizada com critérios de limite de, pelo menos, três ocorrências pelo software VosViewer usando extraído da base de dados. Para cada uma das 78 palavras-chave, a força total dos links de co-ocorrência com outras palavras-chave será calculada. Das 2.530 palavras-chave, 78 cumprem o limiar.

Fonte: Os autores 
Tabela 4 - Outliers 


\begin{tabular}{|c|c|}
\hline Autores & Escopo da Pesquisa \\
\hline Ko; Kirsch; King (2005) & Analisa a transferência de conhecimento, sistemas de informação e comunicação. \\
\hline Kanawattanachai; Yoo (2007) & Transferência de conhecimento em times virtuais \\
\hline $\begin{array}{l}\text { Davies; Green; Rosemann; Indulska; Gallo } \\
\text { (2006) }\end{array}$ & $\begin{array}{l}\text { Análise dos fatores que influenciam a decisão dos analistas, a comunicação para / de pa } \\
\text { (falta de) de técnicas, entre outros. }\end{array}$ \\
\hline Frost; Massagli (2008) & Informação em projetos da área da saúde \\
\hline Boland; Lyytinen; Yoo (2007) & Estudo de projetos de inovação complexos \\
\hline $\begin{array}{l}\text { Akgun; Byrne; Keskin; Lynn; Imamoglu } \\
(2005)\end{array}$ & Armazenamento de informações, memória, em projetos de desenvolvimento de novos \\
\hline Vlaar; van Fenema; Tiwari (2008) & Conhecimento e experiência em Projetos de TI com times geograficamente distribuído \\
\hline $\begin{array}{l}\text { Arnold; Bauersachs; Beier; Blum; Einspanier; } \\
\text { Frohlich; Herrler; Hiendleder; Kolle; PrelleK; } \\
\text { Reichenbach; Stojkovic; Wenigerkind; } \\
\text { Sinowatz (2003) }\end{array}$ & Gestão do conhecimento em projetos genéticos \\
\hline $\begin{array}{l}\text { McKibbon; Lokker; Wilczynski; Ciliska; } \\
\text { Dobbins; Davis; Haynes; Straus (2010) }\end{array}$ & Análise da literatura sobre transferência do conhecimento e barreiras de comunicação \\
\hline Westgate; Likens; Lindenmayer (2013) & Projetos de biodiversidade e ecossistemas \\
\hline Moenaert; Caeldries; Lievens; Wauters (2000) & Requisitos de comunicação que uma equipe internacional de projetos de inovação. \\
\hline Guillery; Harting (2003) & Projetos de tecnologia da área de física e química \\
\hline Bystrom (2002) & Complexidade nas informações e fontes de informação nas tarefas dos projetos \\
\hline Smith; Villalba (2008) & Projetos da área da saúde \\
\hline So; $\operatorname{Kim}(2009)$ & Projetos de tecnologia educacional \\
\hline Wang; Kosakowski; Kolditz (2009) & Projetos de tecnologia da área de física e química \\
\hline Alexander; Godwin (2006) & Projetos da área da saúde \\
\hline He; Butler; King (2007) & $\begin{array}{l}\text { Analisa o surgimento e a evolução da cognição de equipes em equipes de projetos de ss } \\
\text { comunicação e a diversidade de equipes afetam a formação dessas estruturas. }\end{array}$ \\
\hline $\begin{array}{l}\text { Van Boxstael; Habib; Jacxsens; De Vocht; } \\
\text { Baert; Van de Perre; Rajkovic; Lopez-Galvez; } \\
\text { Sampers; Spanoghe; De Meulenaer; } \\
\text { Uyttendaele (2013) }\end{array}$ & $\begin{array}{l}\text { Projeto comercial para captar opiniões de partes interessadas sobre questões de seguran } \\
\text { fornecimento de produtos frescos. }\end{array}$ \\
\hline $\begin{array}{l}\text { van der Sluijs; Petersen; Janssen; Risbey; } \\
\text { Ravetz (2008) }\end{array}$ & Projeto sobre questões de segurança alimentar na cadeia global de fornecimento de pro \\
\hline $\begin{array}{l}\text { Dalgleish; Flicek; Cunningham; Astashyn; } \\
\text { Tully; Proctor; Chen; McLaren; Larsson; } \\
\text { Vaughan; Beroud; Dobson; Lehvaeslaiho; } \\
\text { Taschner; den Dunnen; Devereau; Birney; } \\
\text { Brookes; Maglott (2010) }\end{array}$ & Projetos da área da saúde \\
\hline $\begin{array}{l}\text { Dancet; Van Empel; Rober; Nelen; Kremer; } \\
\text { D'Hooghe (2011) }\end{array}$ & Projetos da área da saúde \\
\hline Barak $(2007)$ & Projetos de tecnologia educacional \\
\hline Kolkman; Kok; van der Veen (2005) & Gestão do Conhecimento em projetos públicos \\
\hline $\begin{array}{l}\text { Sandstrom; Pahlen; Edenius; Tommervik; } \\
\text { Hagner; Hemberg; Olsson; Baer; Stenlund; } \\
\text { Brandt; Egberth (2003) }\end{array}$ & Projetos da área agrícola \\
\hline Park; Lee (2014) & Analisa o papel da dependência e confiança no compartilhamento de conhecimento em \\
\hline Yin; Struik (2008) & Projetos da área da saúde \\
\hline Kleinsmann; Buijs; Valkenburg (2010) & Analisa integração do conhecimento no desenvolvimento colaborativo de novos produt \\
\hline Finlay; Faulkner (2005) & Gestão da comunicação em projetos da área da saúde \\
\hline & $\begin{array}{l}=\text { Total de Citações } \\
=\text { Média de Citações }\end{array}$ \\
\hline
\end{tabular}

Fonte: Os autores 
As palavras-chave estão agrupadas em sete clusters e as ocorrências são apresentadas na Tabela 5. Um mapa de termos demonstra a estrutura de um campo científico, mostrando a relação entre importantes termos da área. Durante a seleção dos termos, são verificados os principais tópicos da área e a relação dos termos com estes, para identificar a sua relevância em diferenciar cada artigo (VAN ECK et al., 2010). 
Os clusters de palavras-chave misturam termos relacionados a comunicação e conhecimento, os termos do cluster \#1 estão mais voltados aos tipos de projetos complexos analisados. $\mathrm{O}$ cluster \#2 apresenta termos voltados para sucesso e desempenho dos projetos. Os termos do cluster \#3 estão relacionados ao compartilhamento do conhecimento, expertise e capacidade do time do projeto. O cluster \#4 mostra assuntos voltados a projetos de tecnologia e construção, o cluster \#5 trabalhos sobre projetos interdisciplinares, o cluster \#6 riscos e informação nos processos de decisão e finalmente o cluster \#7 são apresentados temas voltados para os resultados desenvolvidos pelas pesquisas, como frameworks e perspectivas de trabalhos futuros

Tabela 5 - Ocorrência das palavras-chave

\begin{tabular}{|c|c|c|c|c|c|c|c|}
\hline$\#$ & Cluster 1 & $\#$ & Cluster 2 & $\#$ & Cluster 3 & $\#$ & Cluster 5 \\
\hline 74 & knowledge & 32 & performance & 7 & knowledge transfer & 8 & work \\
\hline 42 & management & 22 & innovation & 6 & expertise & 6 & integration \\
\hline 21 & science & 16 & model & 6 & group-performance & 6 & $\begin{array}{l}\text { transdisciplinary } \\
\text { research }\end{array}$ \\
\hline 19 & complexity & 16 & $\begin{array}{l}\text { project } \\
\text { management }\end{array}$ & 6 & $\begin{array}{l}\text { project } \\
\text { performance }\end{array}$ & 5 & interdisciplinary \\
\hline 13 & education & 13 & $\begin{array}{l}\text { product } \\
\text { development }\end{array}$ & 5 & capacbilities & 5 & projects \\
\hline 13 & impact & 11 & $\begin{array}{l}\text { knwoledge } \\
\text { management }\end{array}$ & 5 & $\begin{array}{l}\text { transactive memory- } \\
\text { system }\end{array}$ & $\#$ & Cluster 6 \\
\hline 13 & implementation & 10 & coordination & $\#$ & 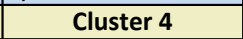 & 19 & information \\
\hline 10 & behavior & 8 & success & 28 & design & 12 & decision-making \\
\hline 10 & health & 7 & case study & 16 & organizarions & 10 & risk \\
\hline 10 & uncertainty & 7 & development teams & 13 & technology & 5 & participation \\
\hline 9 & care & 7 & internet & 11 & construction & 5 & perceptions \\
\hline 9 & project & 7 & $\begin{array}{l}\text { research-and- } \\
\text { development }\end{array}$ & 11 & networks & 5 & power \\
\hline 8 & climate-change & 7 & teams & 7 & system & \# & Cluster 7 \\
\hline 8 & models & 6 & industry & 6 & experience & 19 & perspective \\
\hline 8 & sustainability & 6 & leadership & 6 & simultation & 10 & framework \\
\hline 6 & conservation & $\#$ & Cluster 3 & 6 & teamwork & 5 & issues \\
\hline 5 & ecology & 80 & communication & 5 & communities & & \\
\hline 5 & environment & 14 & trust & 5 & ontology & & \\
\hline 5 & guidelines & 8 & virtual team & $\#$ & $\begin{array}{l}\text { Cluster } 5 \\
\end{array}$ & & \\
\hline 5 & health-care & 7 & $\begin{array}{l}\text { information- } \\
\text { systems }\end{array}$ & 26 & collaboration & & \\
\hline 5 & services & 7 & $\begin{array}{l}\text { information- } \\
\text { technology }\end{array}$ & 25 & systems & & \\
\hline 5 & $\begin{array}{l}\text { social networl } \\
\text { analysis }\end{array}$ & 7 & knowledge sharing & 9 & challenges & & \\
\hline
\end{tabular}

Fonte: Os autores

A Figura 4 apresenta a rede de autores citados que tem pelo menos dois artigos na base de dados selecionada e que foram citados pelo menos duas vezes. Os resultados apresentam 9 artigos, assim o artigo \#ii de Alin, Iorio e Taylor (2013) citaram o artigo \#iv de Boland, Lyytinen e Yoo (2007). O objetivo do artigo \#ii de Alin, Iorio e Taylor (2013) é explorar o papel dos objetos de fronteira digital na negociação de conhecimento de design complexo em 
um espaço de trabalho virtual tridimensional (3-D). Assim os autores Boland, Lyytinen e Yoo (2007) foram citados na seção introdutória do artigo no trecho que afirma ser um desafio o desenvolvimento de projetos em rede.

O artigo \#ii dos autores Dossick et al., (2015) citaram o artigo \#iii de Nayak e Taylor (2009), sendo que o objetivo do artigo \#ii é analisar as equipes de engenharia que colaboram em ambientes virtuais enfrentam muitos problemas técnicos, sociais e culturais, concentrando em equipes distribuídas que fazem descobertas conjuntas imprevistas em ambientes virtuais. Assim, o artigo \#iii do Nayak e Taylor (2009) contribuíram no referencial teórico deste artigo com a definição de times virtuais.

Outra relação encontrada entre os artigos é a do artigo \#vi dos autores Park e Lee (2014) que citaram o artigo \#v de Kanawattanachai e Yoo (2007), onde estes últimos autores auxiliaram, com a teoria apresentada em seus estudos, na construção da hipótese: "A confiança no parceiro está positivamente relacionada ao compartilhamento de conhecimento" do artigo \#vi.

\section{Figura 4 - Citação dos autores}
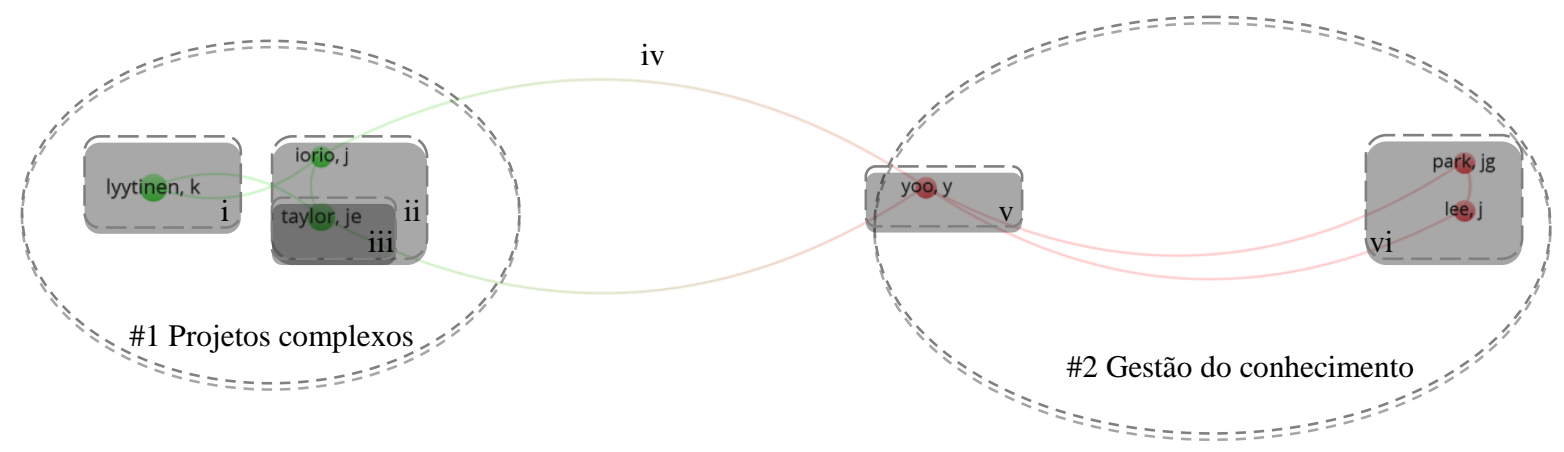

¡ VOSviewer 


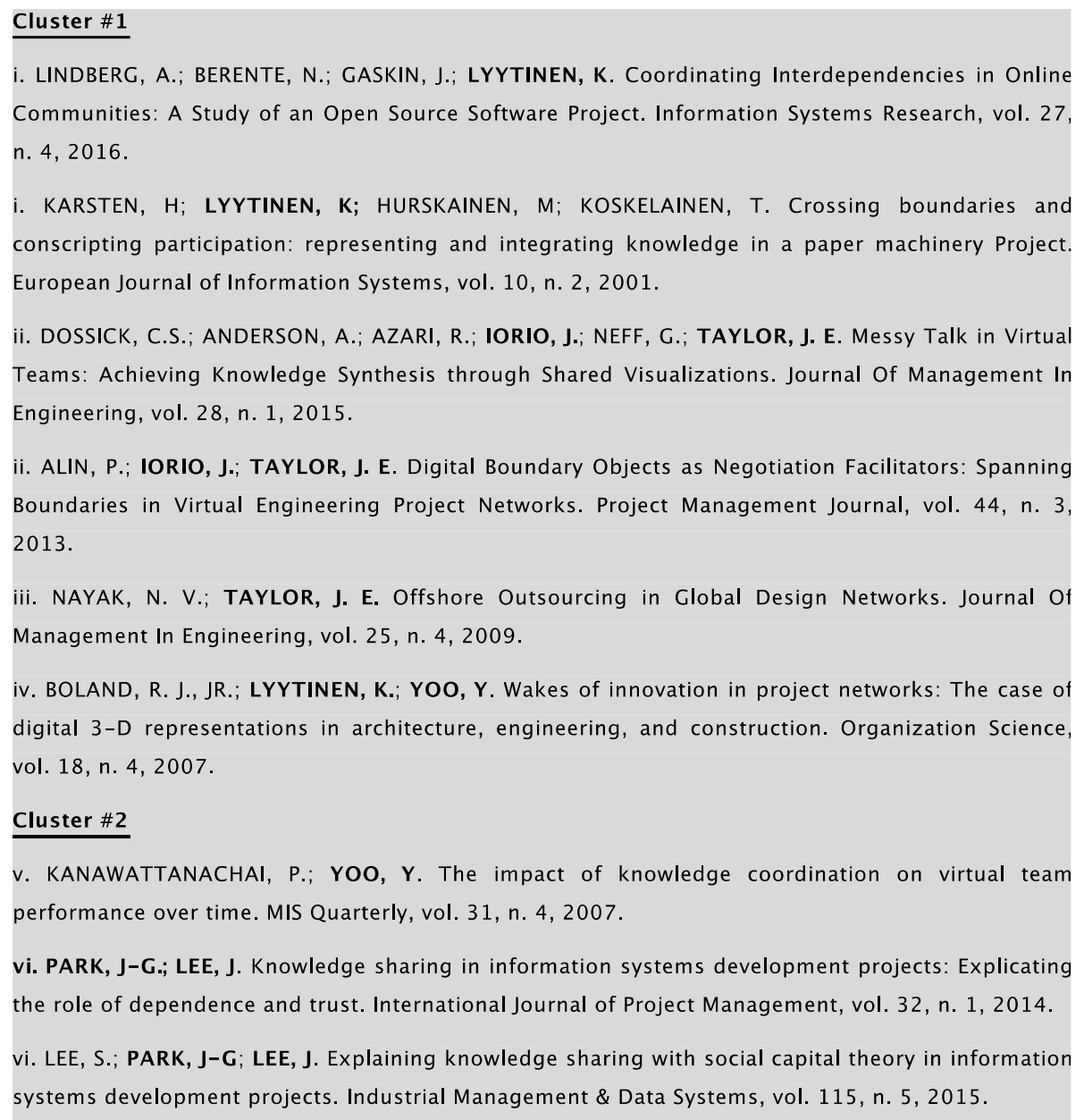

Nota: Esta rede foi realizada com critérios limítrofes de pelo menos 2 citações de uma referência citada pelo software VosViewer usando extraído da base de dados.

Fonte: Os autores

O primeiro cluster apresenta estudos sobre projetos complexos em geral, projetos com times virtuais, TI e inovação. Já os estudos do segundo cluster composto pelos autores Youngjin Yoo, Jun-Gi Park e Jungwoo Lee focam em estudos sobre a gestão do conhecimento, compartilhamento de conhecimento e informação no desenvolvimento de projetos e sua relação com o desempenho dos projetos. Em ambos o artigo mais recente é do ano de 2016.

\section{Conclusão}

O objetivo deste trabalho foi analisar as publicações sobre gestão da comunicação e do conhecimento em projetos complexos, por meio de uma revisão sistemática da literatura e bibliometria. Para a revisão sistemática da literatura buscou-se artigos, utilizando os termos communication* and complex* and project* and knowledge"na base de dados da Web of Science, permitindo analisar os principais autores sobre o tema, tendências de pesquisa e gaps 
na literatura que possibilitarão desenvolvimento de futuras pesquisas que contribuirão com o tema. Além disso, foi possível responder à questão: Como a literatura relaciona gestão da comunicação e gestão do conhecimento em projetos complexos? e Quais são as principais tendências na literatura acadêmica sobre a gestão da comunicação e a gestão do conhecimento em projetos complexos?

Nota-se que a literatura engloba estudos sobre gestão da comunicação nos times dos projetos, mais precisamente sobre armazenagem e compartilhamento do conhecimento em projetos de TI, saúde, física, química, construção, entre outros. Poucos estudos focam em projetos complexos, principalmente analisando os dois tópicos conjuntamente. A gestão da comunicação e a gestão do conhecimento nos projetos complexos, que de acordo com a teoria são projetos com difícil compreensão e previsão, com informações complexas, muitos envolvidos, com interdependência entre projetos, entre outras propriedades que se diferenciam dos projetos, assim, projetos complexos já apresenta desafios diversos o que inclui problemas com comunicação e gestão do conhecimento.

Destaca-se a ausência de artigos, na base utilizada, que analisam as barreiras à comunicação em projetos e barreiras à gestão do conhecimento em projetos, principalmente analisando estas barreiras nos projetos complexos, não foram encontrados estudos que fazem esta relação. Assim, com base nos artigos analisados existem poucos estudos que relacionam gestão da comunicação e gestão do conhecimento em projetos e nenhum artigo que relaciona gestão da comunicação e gestão do conhecimento em projetos complexos. Individualmente os temas são estudados por diversos autores, como apresentados nas tabelas e analises deste estudo.

Os artigos do ano de 2017 em sua maioria tratam dos temas de conhecimento e aprendizagem, confiança e comunicação, projetos complexos em TI e na área da saúde, sustentabilidade em projetos e a influência do conhecimento e da comunicação nas mudanças ambientais dos projetos. E os artigos mais recentes do ano de 2018 tratam da análise da aprendizagem em projetos da área de saúde, comunicação em projetos sustentáveis, conhecimento e comunicação interpessoal em projetos educacionais, conhecimento de política em megaprojetos.

Futuras pesquisas poderão analisar gestão da comunicação e gestão do conhecimento em projetos complexos; as barreiras e principais desafios dos projetos complexos; as diferenças entre a gestão da comunicação em projetos tradicionais e projetos complexos e o mesmo para gestão do conhecimento. 
Este estudo limita-se a analisar apenas os artigos da base de dados da Web of Science, outras bases poderiam ser incorporadas e analisadas, como também artigos de outros idiomas.

\section{Referências}

ALLEN, P. M. The importance of complexity for the research agenda in the built environment. Architectural Engineering and Design Management, v. 4, n. 1, p. 5-14, 2008.

ALONSO, L. B. N. et al. Comunicação e compartilhamento do conhecimento entre equipes em automação de processos. Revista Comunicologia, n. 2002, p. 165-183, 2013.

BACCARINI, D. The concept of project complexity - A review. International Journal of Project Management, v. 14, n. 4, p. 201-204, 1996.

BANO, M.; ZOWGHI, D.; SARKISSIAN, N. Empirical study of communication structures and barriers in geographically distributed teams. IET Software, v. 10, n. 5, p. 147-153, 2016.

BENBYA, H.; MCKELVEY, B. Toward a complexity theory of information systems development. Information Technology \& People, v. 19, n. 1, p. 12-34, 2006.

CARVALHO, M. M.; PATAH, L. A.; BIDO, D. DE S. Project management and its effects on project success: Cross-country and cross-industry comparisons. International Journal of Project Management, v. 33, n. 7, p. 1509-1522, 2015.

CARVAlHO, M. M.; RABECHINI JR, R. Fundamentos em Gestão de Projetos: Construindo Competências para Gerenciar Projetos. Atlas ed. São Paulo: 4, 2015.

CHAPMAN, R.; HYLAND, P. Complexity and learning behaviors in product innovation. Technovation, v. 24, n. 7, p. 553-561, 2004.

CICMIL, S.; MARSHALL, D. Insights into collaboration at the project level: Complexity, social interaction and procurement mechanisms. Building Research and Information, v. 33, n. 6, p. 523- 535, 2005.

COOKE-DAVIES, T. et al. We're Not in Kansas Anymore, Toto: Mapping the Strange Landscape of Complexity Theory, and Its Relationship to Project Management. IEEE Engineering Management Review, v. 36, n. 2, p. 5-21, 2008.

CRAWFORD, L.; HOBBS, B.; TURNER, J. . Project Categorization Systems. Newton Square: PMI, 2005.

FIGUEIRA, M. M. C. Identificação de outliers. 1998. Disponível em: <http://www.ipv.pt/millenium/arq12.htm>. Acesso em: 10 out. 2017.

FUKUYAMA, K. et al. Barreiras à comunicação e suas influências no desempenho de projeto. Mundo PM, p. 1-56, 2015.

GERALDI, J.; ADLBRECHT, G. On faith, fact, and interaction in projects. IEEE Engineering Management Review, v. 36, n. 2, p. 35-49, 2007.

GERALDI, J.; MAYLOR, H.; WILLIAMS, T. Now, let's make it really complex (complicated). International Journal of Operations \& Production Management, v. 31, n. 9, p. 966-990, 2011. GIRMSCHEID, G.; BROCKMANN, C. The Inherent Complexity of Large Scale Engineering Projects. Project Perspectives, v. 29, p. 22-26, 2008.

GREEN, G. C. The impact of cognitive complexity on project leadership performance. Information and Software Technology, v. 46, p. 165-172, 2004.

HE, Q. et al. Measuring the complexity of mega construction projects in China-A fuzzy analytic network process analysis. International Journal of Project Management, v. 33, n. 3, p. 549-563, 2015.

HOBDAY, M. Product complexity, innovation and industrial organisation. Research Policy, v. 26, n. 6, p. 689-710, 1998. 
HOMER-DIXON, T. F. The ingenuity gap: Facing the economic, environmental, and other challenges of an increasingly complex and unpredictable world. New York.: Knopf, 2000.

IPMA - INTERNACIONAL PROJECT MANAGEMENT ASSOCIATION. ICB - IPMA Competence Baseline, Version 3.0. [s.l: s.n.].

JOHANNESSEN, J. A.; OLSEN, B. Projects as communicating systems: Creating a culture of innovation and performance. International Journal of Information Management, v. 31, n. 1, p. 3037, 2011.

JOHANSEN, J.; GILLARD, S. Information resources project management communication: Personal and environmental barriers. Journal of Information Science, v. 31, n. 2, p. 91-98, 2005.

KIM, J.; WILEMON, D. Sources and assessment of complexity in NPD projects. R and D Management, v. 33, n. 1, p. 15-30, 2003.

LEE, J. N. The impact of knowledge sharing, organizational capability and partnership quality on IS outsourcing success. Information and Management, v. 38, n. 5, p. 323-335, 2001.

LITTLE, T. Uncertainty. IEEE Computer Society, p. 28-35, 2005.

LUO, L. et al. Investigating the Relationship between Project Complexity and Success in Complex Construction Projects. Journal of Management in Engineering, v. 33, n. 2, p. 4016036, 2017.

MARKS, M. A.; MATHIEU, J. E.; ZACCARO, S. J. A Temporally Based Framework and Taxonomy of Team Processes Author ( s ): Michelle A . Marks, John E . Mathieu and Stephen J . Zaccaro Source: The Academy of Management Review, Vol . 26 , No . 3 ( Jul ., 2001 ), pp . 356-376 Published by: Academy of M. The Academy of Management Review, v. 26, n. 3, p. 356-376, 2001.

MATTAR, F. N. Pesquisa de Marketing: Metodologia, planejamento, execução e análise. 7. ed. Rio de Janeiro: Elsevier. 2014.

MÜLLER, R.; TURNER, J. R. Matching the project manager's leadership style to project type. International Journal of Project Management, v. 25, n. 1, p. 21-32, 2007.

MYKYTYN, P. P.; GREEN, G. I. Effects of computer experience and task complexity on attitudes of managers. Information and Management, v. 23, n. 5, p. 263-278, 1992.

RABECHINI JR., R.; CARVALHO, M. M. DE; LAURINDO, F. J. B. Fatores críticos para implementação de gerenciamento por projetos: o caso de uma organização de pesquisa. Produção, v. 12, n. 2, p. 28-41, 2002.

SANTOS, V. R. et al. Knowledge Sharing Barriers in Complex Research and Development Projects: an Exploratory Study on the Perceptions of Project. Knowledge and Process Management, v. 23, n. 2, p. 110-123, 2016.

SHENHAR, A. J. One size does not fit all projects: exploring classical contingency domains. Management Science, v. 47, n. 3, p. 394-414, 2001

SHENHAR, A. J.; DVIR, D. Reinventing Project Management: The Diamond Approach to Successful Growth and Innovation. Boston, MA: HBS Press Book, 2007.

SHENHAR, A. J.; DVIR, D.; SHULMAN, Y. A two-dimensional taxonomy of products and innovations. Journal of Engineering and Technology Management, v. 12, n. 3, p. 175-200, 1995.

TATIKONDA, M. V.; ROSENTHAL, S. R. Technology novelty, project complexity, and product development project execution success: A deeper look at task uncertainty in product innovation. HPAC Heating, Piping, Air Conditioning, v. 72, n. 2, p. 74-87, 2000.

VAN ECK, N. J., WALTMAN, L. Software survey: VOSviewer, a computer program for bibliometric mapping. Scientometrics, v. 84, p. 523-538, 2010.

VAN ECK, N. J., WALTMAN, L., DEKKER, R., VAN DEN BERG, J. A. Comparison of Two Techniques for Bibliometric Mapping: Multidimensional Scaling and VOS. Journal of the American Society for Information Science and Technology, v. 61, p. 2.405-2.416, 2010.

VIDAL, L.; MARLE, F. Understanding project complexity: implications on project management. Kybernetes, v. 37, n. 8, p. 1094-1110, 2008. 
WILLIAMS, T. Assessing and moving on from the dominant project management discourse in the light of project overruns. IEEE Transactions on Engineering Management, v. 52, n. 4, p. 497-508, 2005.

WILLIAMS, T. M. The need for new paradigms for complex projects. International Journal of Project Management, v. 17, n. 5, p. 269-273, 1999.

WILliams, T. Modelling Complex Projects. [s.1.] Wiley, Chichester, 2002.

WILliAMS, T. Post-project reviews. Newtown Square, Pennsylvania: Project Management Institute, Inc, 2007.

XIA, W. Complexity of Information Systems Development Projects: Conceptualization and Measurement Development Complexity of Information Systems Development Projects: Conceptualization and

Measurement Development Abstract. Journal of Management Information Systems, v. 22, n. 1, p. 4583, 2005. 